11

\title{
Флуктуационный анализ динамики систем с меняющимися во времени характеристиками
}

\author{
(C) О.Н. Павлова, А.Н. Павлов \\ Саратовский национальный исследовательский государственный университет им. Н.Г. Чернышевского, \\ Саратов, Россия \\ ฯE-mail: pavlov.alexeyn@gmail.com
}

Поступило в Редакцию 10 декабря 2020г.

В окончательной редакции 10 декабря 2020 г.

Принято к публикации 17 февраля 2021 г.

Проводится модернизация метода флуктуационного анализа, включающая учет статистики локальных стандартных отклонений профиля сигнала от кусочно-линейной аппроксимации тренда. Показано, что предлагаемый подход позволяет снизить чувствительность метода к отдельным артефактам и повысить устойчивость алгоритма вычисления показателя скейлинга, что способствует более широкому использованию модернизированного флуктуационного анализа для решения задач диагностики сложных процессов в динамике систем с меняющимися во времени характеристиками.

Ключевые слова: флуктуационный анализ, случайный процесс, нестационарность, показатель скейлинга.

DOI: 10.21883/PJTF.2021.09.50910.18653

Флуктуационный анализ, предусматривающий процедуру аппроксимации и удаления низкочастотной динамики или тренда (detrended fluctuation analysis, DFA) [1,2], является полезной альтернативой классическому корреляционному анализу. В отличие от автокорреляционной функции он обеспечивает более надежное оценивание характеристик дальних корреляций, особенно при наличии помех и в условиях нестационарности. Несмотря на то что для стационарных случайных процессов существует взаимосвязь между показателем скейлинга метода DFA и характеристиками, описывающими спад автокорреляционной функции или частотную зависимость функции спектральной плотности мощности [1], наличие более универсального подхода, применимого как к стационарным, так и к нестационарным процессам, послужило причиной широкого использования метода DFA в исследованиях динамики сложных систем по экспериментальным данным [3-10]. Как и любой другой метод цифровой обработки сигналов, DFA имеет свои ограничения, которые обсуждались, например, в работах [11-14]. В [15] показано, что различные типы нестационарности (тренд, перемежающееся поведение, изменение во времени энергетических характеристик) оказывают влияние на результаты метода DFA и могут привести к их неправильной интерпретации. По этой причине сведение сигнала к стационарности на этапе предварительной обработки (если это возможно) является необходимой процедурой.

В условиях сильного изменения характеристик динамики системы во времени, например при переходных процессах, когда свойства исследуемого сигнала существенно различаются на разных участках, нами было предложено использовать модифицированный метод DFA [16], предусматривающий расчет дополнительного показателя скейлинга, который характеризует эффекты нестационарности. Этот модифицированный подход учитывает различия локальных среднеквадратичных отклонений профиля сигнала от кусочно-линейной аппроксимации тренда. В настоящей работе предложена дальнейшая модернизация метода для обеспечения более стабильных результатов анализа.

Алгоритм метода DFA [2] включает переход от сигнала $x(i), i=1, \ldots, N$, к его профилю в рамках обобщенной модели одномерных случайных блужданий

$$
Y(k)=\sum_{i=1}^{k}[x(i)-\langle x\rangle], \quad\langle x\rangle=\sum_{i=1}^{N} x(i), \quad k=1, \ldots, N,
$$

сегментацию профиля $Y(k)$ на неперекрывающиеся участки длины $n$ и линейную аппроксимацию тренда $Y_{n}(k)$ в каждом участке. Далее вычисляется стандартное отклонение флуктуаций профиля относительно тренда

$$
F(n)=\sqrt{\frac{1}{N} \sum_{k=1}^{N}\left[Y(k)-Y_{n}(k)\right]^{2}},
$$

и аналогичные расчеты проводятся в широком диапазоне $n$ для анализа степенного поведения зависимости $F(n)$ и оценки показателя скейлинга $\alpha$

$$
F(n) \sim n^{\alpha}
$$

Такое поведение характерно для многих случайных процессов, хотя значение $\alpha$ может различаться в разных диапазонах масштабов.

Если характеристики нестационарного поведения сильно варьируются во времени (например, для переходных процессов или режимов перемежаемости), то 
эти изменения влияют на зависимость (3). Для такого случая в работе [16] предложено учитывать локальные стандартные отклонения профиля от линейной аппроксимации $F_{l o c}(n)$, вычисляемые для каждого сегмента в отдельности. Эффекты нестационарности можно охарактеризовать с помощью меры

$$
d F(n)=\max \left[F_{l o c}(n)\right]-\min \left[F_{l o c}(n)\right],
$$

которая принимает малые значения для однородного процесса и возрастает, если свойства нестационарности меняются в зависимости от сегмента исходных данных.

Для меры (4) характерен рост при увеличении $n$, однако степенное поведение $d F(n)$ описывается показателем скейлинга $\beta$

$$
d F(n) \sim n^{\beta},
$$

который в общем случае отличен от $\alpha$. Такой вариант модификации метода DFA продемонстрировал возможность улучшения диагностики структурных изменений сигналов по сравнению со стандартным алгоритмом при анализе физиологических процессов [17]. Однако он имеет существенный недостаток: появляется чувствительность к одиночным артефактам, влияющим на величину $\max \left[F_{l o c}(n)\right]$ и, следовательно, на показатель скейлинга. Чтобы избежать этого и повысить устойчивость алгоритма, целесообразно использовать статистические характеристики, например вычислять среднеквадратичное отклонение локальных значений $F_{l o c}(n)$, т.е. анализировать зависимость

$$
\sigma\left(F_{l o c}(n)\right) \sim n^{\beta}
$$

$\mathrm{C}$ учетом различий в определениях показатель $\beta$ может отличаться при использовании формул (5) и (6). В данном случае мы выбираем только один вариант расчета, который обеспечивает более стабильные результаты. В соответствии с данными рис. 1 для случайного процесса с антикорреляциями $(\alpha \approx 0.04$, рис. $1, a)$ незначительный рост $\lg F(n)$ с увеличением размера сегмента сопровождается спадом значений $\lg d F$ и $\lg \sigma\left(F_{l o c}\right)$, т.е. отрицательным показателем скейлинга $\beta$. При этом разброс значений относительно аппроксимирующей прямой меньше при рассмотрении зависимости (6) вместо (5). Для белого шума $(\alpha=0.5$, рис. $1, b)$ и режима перемежаемости между этими случайными процессами (рис. 1,c) наблюдается примерное соответствие показателей скейлинга $\alpha$ и $\beta$, но для более стабильных расчетов также лучше использовать $\sigma\left(F_{l o c}(n)\right)$ вместо $d F(n)$.

На рис. 1 приведены примеры анализа сигналов, показатели скейлинга которых сохраняются при изменении масштаба. В качестве примера неоднородного процесса, который демонстрирует различные показатели скейлинга для ближних и дальних корреляций, был рассмотрен четырехчасовой сигнал электроэнцефалограммы крысы, включающий участки бодрствования и медленной фазы сна. На рис. 2 приведены зависимости, описываемые формулами (3), (5) и (6), в двойном логарифмическом
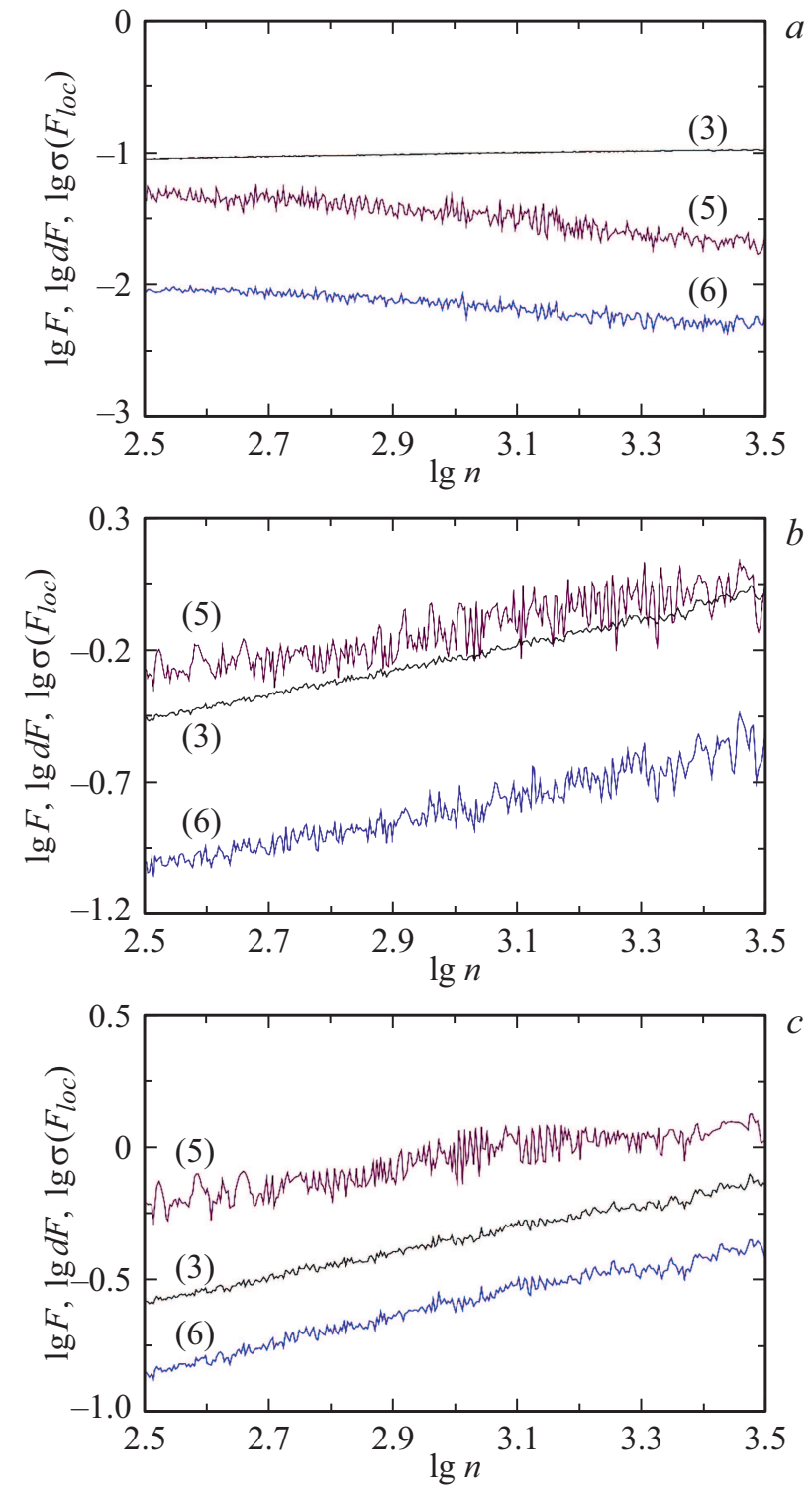

Рис. 1. Зависимости (3), (5) и (6), представленные в двойном логарифмическом масштабе, для тестовых сигналов - антикоррелированного случайного процесса (производной $1 / f$-шума) $(a)$, белого шума $(b)$ и периодических переключений между этими процессами $(c)$.

масштабе. В диапазоне $2.5<\lg n<3.5$ наклоны графиков достаточно близки, однако для дальних корреляций $(\lg n>4.0)$ поведение принципиально меняется, и положительному $\alpha$ соответствует отрицательный показатель $\beta$. Во всех диапазонах масштабов использование среднеквадратичных отклонений величин $F_{l o c}(n)$ вместо разности экстремальных величин (4) обеспечивает снижение разброса вычисленных значений. Полученные результаты подтверждают независимость показателей скейлинга модифицированного метода DFA [16]. Кроме того, они свидетельствуют о том, что предлагаемая в работе дальнейшая модернизация метода, использующая статистику локальных стандартных отклонений профиля 


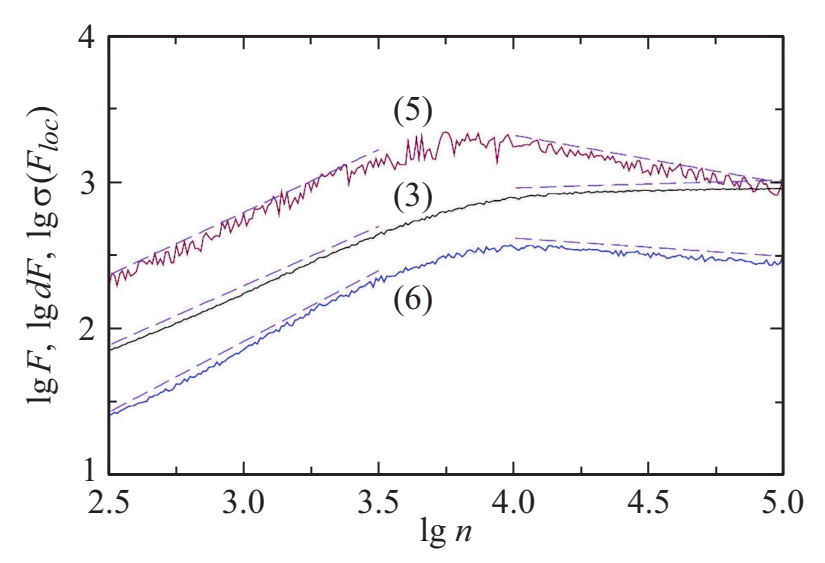

Рис. 2. Зависимости (3), (5) и (6), представленные в двойном логарифмическом масштабе, для сигнала электроэнцефалограммы крысы (частота дискретизации $2 \mathrm{kHz}$ ).

сигнала от линейной аппроксимации тренда, позволяет обеспечить более стабильные результаты вычислений, которые достигаются за счет уменьшения разброса значений $\lg \sigma\left(F_{l o c}\right)$ по сравнению с $\lg d F$ при анализе степенных закономерностей, описываемых формулами (5) и (6). Данное обстоятельство целесообразно учитывать при использовании модифицированного метода DFA для решения задач диагностики сложных процессов в динамике систем с меняющимися во времени характеристиками.

\section{Финансирование работы}

Исследование выполнено при поддержке гранта Президента РФ для государственной поддержки ведущих научных школ РФ (НШ-2594.2020.2) и математического центра СГУ им. Н.Г. Чернышевского.

\section{Соблюдение этических стандартов}

Все применимые международные, национальные и/или институциональные принципы ухода и использования животных были соблюдены.

\section{Конфликт интересов}

Авторы заявляют, что у них нет конфликта интересов.

\section{Список литературы}

[1] C.-K. Peng, S.V. Buldyrev, S. Havlin, M. Simons, H.E. Stanley, A.L. Goldberger, Phys. Rev. E, 49 (2), 1685 (1994). DOI: 10.1103/PhysRevE.49.1685

[2] C.-K. Peng, S. Havlin, H.E. Stanley, A.L. Goldberger, Chaos, 5 (1), 82 (1995). DOI: 10.1063/1.166141

[3] K. Kiyono, Y. Tsujimoto, Physica A, 462, 807 (2016). DOI: $10.1016 /$ j.physa.2016.06.129
[4] G. Bhoumik, A. Deb, S. Bhattacharyya, D. Ghosh, Adv. High Energy Phys., 2016, 7287803 (2016). DOI: $10.1155 / 2016 / 7287803$

[5] O. Løvsletten, Phys. Rev. E, 96 (1), 012141 (2017). DOI: 10.1103/PhysRevE.96.012141

[6] N.A. Kuznetsov, C.K. Rhea, PLoS ONE, 12 (3), e0174144 (2017). DOI: 10.1371/journal.pone.0174144

[7] G. Nolte, M. Aburidi, A.K. Engel, Sci. Rep., 9, 6339 (2019). DOI: $10.1038 / \mathrm{s} 41598-019-42732-7$

[8] N.S. Frolov, V.V. Grubov, V.A. Maksimenko, A. Lüttjohann, V.V. Makarov, A.N. Pavlov, E. Sitnikova, A.N. Pisarchik, J. Kurths, A.E. Hramov, Sci. Rep., 9, 7243 (2019). DOI: $10.1038 / \mathrm{s} 41598-019-43619-3$

[9] А.Н. Павлов, А.Е. Руннова, В.А. Максименко, О.Н. Павлова, Д.С. Гришина, А.Е. Храмов, Письма в ЖТФ, 45 (4), 8 (2019). DOI: 10.21883/PJTF.2019.04.47327.17377

[10] О.Н. Павлова, А.Н. Павлов, Письма в ЖТФ, 45 (18), 6 (2019). DOI: 10.21883/PJTF.2019.18.48228.17882

[11] K. Hu, P.C. Ivanov, Z. Chen, P. Carpena, H.E. Stanley, Phys. Rev. E, 64 (1), 011114 (2001). DOI: 10.1103/PhysRevE.64.011114

[12] Z. Chen, P.C. Ivanov, K. Hu, H.E. Stanley, Phys. Rev. E, 65 (4), 041107 (2002). DOI: 10.1103/PhysRevE.65.041107

[13] R.M. Bryce, K.B. Sprague, Sci. Rep., 2, 315 (2012). DOI: $10.1038 /$ srep00315

[14] Y.H. Shao, G.F. Gu, Z.Q. Jiang, W.X. Zhou, D. Sornette, Sci. Rep., 2, 835 (2012). DOI: 10.1038/srep00835

[15] A.N. Pavlov, O.N. Pavlova, O.V. Semyachkina-Glushkovskaya, J. Kurths, Eur. Phys. J. Plus., 136, 10 (2021). DOI: $10.1140 /$ epjp/s13360-020-00980-x

[16] А.Н. Павлов, О.Н. Павлова, А.А. Короновский (мл.), Письма в ЖТФ, 46 (6), 47 (2020). DOI: 10.21883/PJTF.2020.06.49166.18136

[17] A.N. Pavlov, A.S. Abdurashitov, A.A. Koronovskii, Jr., O.N. Pavlova, O.V. Semyachkina-Glushkovskaya, J. Kurths, Commun. Nonlinear Sci. Numer. Simulat., 85, 105232 (2020). DOI: $10.1016 /$ j.cnsns.2020.105232 